\title{
RESEARCH
}

Open Access

\section{Associating conditional cash transfer to universal access to treatment could be the solution to the HCV epidemic among drug users (DUs)}

Philippe Chossegros ${ }^{1 *}$ (D) and Fiorant Di Nino ${ }^{2}$

\begin{abstract}
Background: To understand the limits of HCV screening programs to reach all drug users (DUs).

Method: The association of the recruitment of a representative sample of a population of DUs in a specific area with the use of a questionnaire that included 250 items allowed the use of uni- and multifactorial analysis to explore the relationship between HCV screening and dimensions until now restricted to qualitative studies.

Results: We recruited, in less than 2 months, 327 DUs representing about $6 \%$ of the total population of DUs. They belonged to a single community whose drug use was the only common characteristic. While almost all DUs (92.6\%) who had access to care providers had been screened, this proportion was much lower in out-of-care settings (64\%). HCV prevalence among those who had performed a test was low (22.8\%). For DUs, the life experience of hepatitis $C$ has not changed in the last 10 years. Screening, studied for the first time according to this life experience, was not influenced by a rational knowledge of the risk taken or the knowledge of treatment efficacy, showing a gap between DUs' representations and medical recommendations which explains the low level of active screening. Police crackdown on injections, disrupting the previous illusion of safe practices, was the only prior history leading to active screenings. Screenings were related to an access to care providers. GPs held a preponderant position as a source of information and care by being able to give appropriate answers regarding hepatitis C and prescribing opioid substitution treatments (OST). If $48 \%$ of DUs screened positive for HCV had been treated, half of them had been prescribed before 2006.

Conclusion: While hepatitis has become a major issue for society and, consequently, for services for DUs (SDUs) and GPs, it is not the case for DUs. A widespread screening, even in a city where the offer of care is diversified and free, seems unlikely to reach a universal HCV screening over a short time. The model of respondent-driven sampling recruitment could be a new approach to conditional cash transfer, recruiting and treating DUs who remain outside the reach of care providers, a prerequisite for the universal access to HCV treatments to impact the HCV epidemic.
\end{abstract}

Keywords: Drug use, Respondent-driven sampling, HCV, Epidemic, Social networks

\footnotetext{
* Correspondence: philippe.chossegros@chu-lyon.fr

${ }^{1}$ UHSI de Lyon, Centre Hospitalier Lyon SUD, Hospices Civils de LYON,

Chemin du Grand Revoyet, 69495 Pierre-Bénite, France

Full list of author information is available at the end of the article
}

(c) The Author(s). 2018 Open Access This article is distributed under the terms of the Creative Commons Attribution 4.0 International License (http://creativecommons.org/licenses/by/4.0/), which permits unrestricted use, distribution, and reproduction in any medium, provided you give appropriate credit to the original author(s) and the source, provide a link to the Creative Commons license, and indicate if changes were made. The Creative Commons Public Domain Dedication waiver (http://creativecommons.org/publicdomain/zero/1.0/) applies to the data made available in this article, unless otherwise stated. 


\section{Background}

Viral infections of DUs and more particularly hepatitis C [1] are recognized as a public health problem. Hepatitis B vaccination prevents new $\mathrm{HBV}$ infections [2], antiviral treatments of hepatitis B [3, 4] and AIDS [5] control the evolution of these diseases, and direct-acting antivirals (DAA) make it possible to cure almost all hepatitis C [6]. Despite their effectiveness, their impact on the course of these epidemics will not be fully realized as long as patients are left untreated $[7,8]$. A universal screening is a prerequisite to succeed [9].

Due to the illegal nature of drug use, part of the population of DUs remains hidden. Understanding the course of this epidemic suffers from variation in the coverage and quality of existing research and, most of all, of convenience sampling which may be responsible for representative bias $[10,11]$. This lack of reliable data has been emphasized by most international reviews [12-14] as well as French recommendations [15]. More precise data on coherent territories are needed. Different approaches have been proposed to recruit a representative sample of DUs active in a given territory.

Apart from the use of drugs, DUs differ from the general population by their socio-economic situation and by their particularly high risks of contamination by infectious agents. Although $\mathrm{HCV}$ prevalence has been frequently studied, other elements such as the influence of their use of drugs, their social networks, their fears, their projects, their sources of information, and their opioid substitution treatment on their access to screening have been little studied. In 2008, Treloar and Rhodes, reviewing 31 former papers in a qualitative meta-ethnographic analysis of the social production of hepatitis risk, concluded that, for injecting DUs, HCV hepatitis is a liminal illness, a risk accepted rather than avoided and that symbolic knowledge systems, rather than biomedical risk calculus, and especially narratives of hygiene and trust, played a primary role in shaping interpretations of HCV risk [16]. However, non-injectors were excluded, and they did not analyze the relationship of this life experience with HCV screening. The most recent studies were only conducted among intravenous DUs (IVDUs) attending primary and secondary care services. Strauss et al. found a low knowledge of hepatitis $\mathrm{C}$ among injectors and non-injectors who were unaware of the existence of $\mathrm{HCV}$ education opportunities [17]. Barocas et al., in Southern Wisconsin, found that access to healthcare was an important determinant of regular $\mathrm{HCV}$ screening, indicating that self-initiated screening was less successful than provider-initiated HCV screening, consistent with a previous study conducted in San Francisco and New York $[18,19]$. For Swan et al., $\mathrm{HCV}$ was considered as a relatively benign infection, competing with other priorities. Difficulties in accessing specialized care and treatment were improved by trust in providers and education on HCV infection [20]. Walley et al. "interested" attendants of a methadone clinic in $\mathrm{HCV}$ treatment after an education program [21].

In 2011, we decided to improve the quality of our care by a better knowledge of DUs. First, in a partnership between professionals and DUs attending services for DUs (SDUs), we wrote a questionnaire including all relevant information that would help to understand DU's life (Table 1). Then, the same year, we used it to study DUs recruited by a snowball sampling initiated in all the SDUs of the greater Strasbourg. Nobody was paid. If the content of the questionnaire has been validated by its analysis, the snowball recruitment was a failure, since it was only able to recruit 145 DUs for a period of 1 year with 2 DUs outside of the health care system. This failure led us to consider another method to recruit a representative sample of Strasbourg's population of DUs.

Respondent-driven sampling (RDS) is currently one of the most popular. It has been applied in more than 460 peer-reviewed surveys conducted in 69 countries throughout the world [22]. It is used by the Centers for Disease Control and Prevention to track the HIV epidemic [23, 24]. Yet, studies that have applied this method to DUs have been almost constantly limited to risk behaviors, search for viral prevalence, and access to care.

This RDS study was funded in 2015 through a grant of the "Fonds de dotation Gilead Sciences."

We present the analysis of this database from the perspective of $\mathrm{HCV}$ screening, contamination, and treatment. To our knowledge, it is the first study to focus, in a representative sample of DUs in a well-defined area, on the relation between HCV screening history and the elements that may have influenced this past decision or that could lead to further screening and treatment, information that could help build a more effective strategy to eradicate hepatitis $\mathrm{C}$ among DUs.

\section{Methods \\ Study design}

Our study was conducted in the greater Strasbourg area (15-64 years, 473,375 people). RDS was chosen to recruit a representative sample of DUs. The condition of their eligibility followed the European Monitoring Centre for Drugs and Drug Addiction definition of problematic drug use: injecting drug use or long duration/regular use of opioids, cocaine, and/or amphetamines [25]. Non-injectors were included because most injectors begin as non-injectors, because HCV prevalence in this population has been shown to be much higher than in a non-drug using population [26], and because we wanted to compare non-injectors to injectors in their relation to screening. Strauss et al. considered 
Table 1 Items covered in the questionnaire

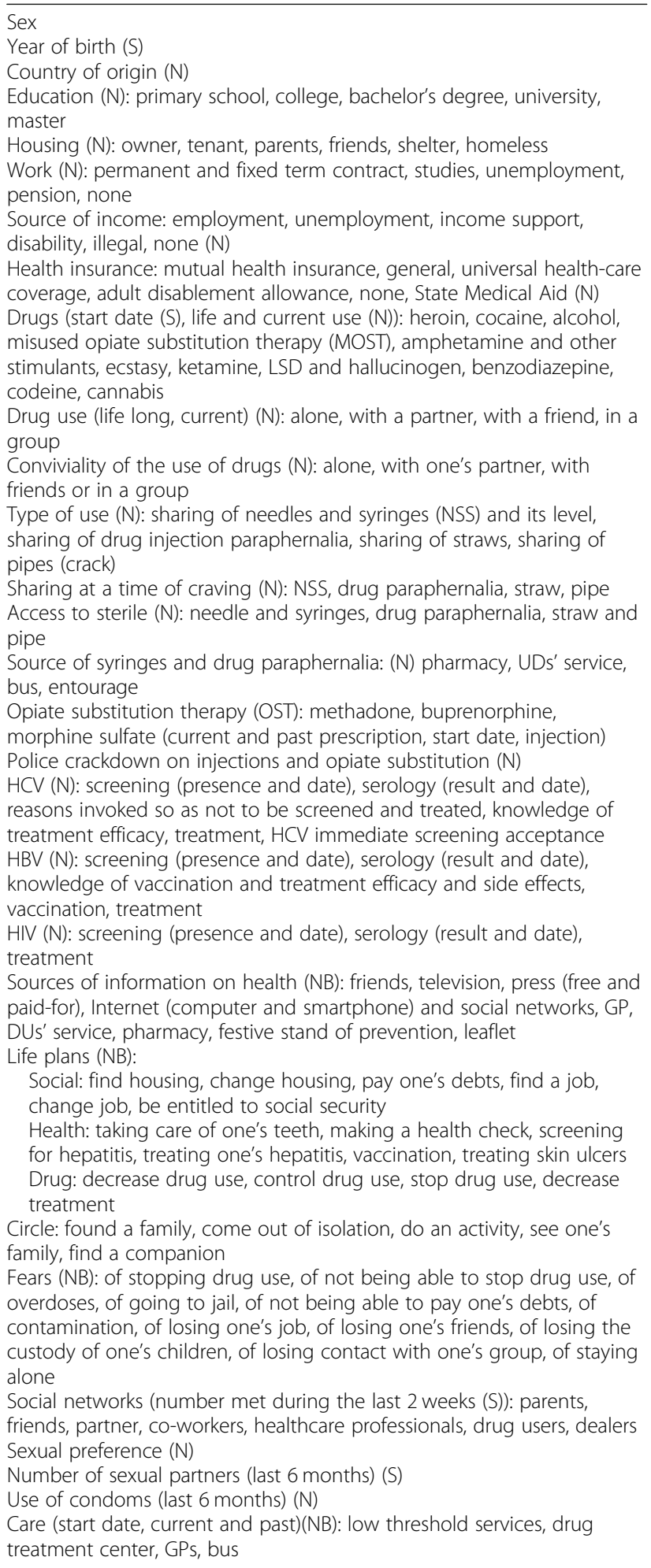

$S$ scale, $N$ nominal, $N B$ nominal binary

critical for prevention that non-injectors understand the $\mathrm{HCV}$ related risks involved in sharing both injection and non-injection drug use equipment [17].
We started with 3 "seeds" randomly recruited among DUs attending the same SDU. They were asked the number of DUs they had met in the previous 14 days (network size) and were given 3 coupons to recruit 3 respondents among their own social network who were due to show up less than 14 days later. Each wave of respondents, following the same rules, recruited the next wave until the final sample size was achieved. Our budget allowed financing the recruitment of 300 DUs representing a sample of $6 \%$ of the total population of UDs of the greater Strasbourg area [27].

The completion of a 250-item auto-questionnaire by each respondent was followed by a cross-examination of their answers in a private space by the same professional interviewer to assessed their quality and completeness. $\mathrm{HCV}$ serostatus was categorized according to participants' self-report. No one had to perform a new HCV screening. The monetary recruitment incentive was $30 €$ for the completion of the interviewer-administered questionnaire and $20 €$ for each recruited UD. This amount was considered to be a fair salary, recognizing an actual work, by the DUs who had validated the questionnaire. Since giving money unconditionally regarding its use to homeless and other low-income individuals, among which DUs, has constantly been shown to improve their empowerment, this approach was considered ethical [28-30]. The conduct of the study was in line with the ethical checklist (respect, beneficence, justice, and safeguards) provided by Semaan and al. [31].

Patients provided informed consent to participate in the study and to publish its results. This study was approved by the French Commission Nationale de l'Informatique et des Libertés (CNIL).

\section{Statistical analysis}

Data were anonymized before analysis. RDS allows statistical adjustments for differential network sizes to produce estimates representative of the sampled population's network. RDS data weighting was used to compare the observed distribution of HCV screening between the observed and the weighted population as well as their homophily for sex and age using RDSAT version 7.1.46: (http://www.respondentdrivensampling.org/reports/RDSA T_7.1-Manual_2012-11-25.pdf; http://www.respondentdrivensampling.org/download/RDSATv7.1.46/RDSAT_windows-x64_7_1_46.exe). Equilibrium was calculated for participant's age, sex, injection, HCV screening, and HCV prevalence.

The items considered pertinent to understand $\mathrm{HCV}$ screening (HCV screening, HCV contamination, knowledge of the efficacy of HCV treatment, plans for the future, fear of contamination) were compared to all the other variables. For ordinal or categorical variables, chi-square and odds ratio or Kendall tau-b were 
calculated. $t$ test or one-way ANOVA was performed for scale variables. Since multiple comparisons were performed, statistical significance was considered at an alpha of 0.01 to prevent the occurrence of too many statistically but not clinically relevant associations. To measure the association between dependent and independent variables, the significant variables were entered into binary logistic regression multivariate models and automatically chosen by forward stepwise selection procedure (two sides, $p<0.05)$. Odds ratios (OR) and $95 \%$ confidence intervals (CI) were calculated from $\beta$ coefficients and their standard errors. The Hosmer-Lemshow test was used to determine the goodness of fit of the models. The data was analyzed using IBM SPSS version 19.

\section{Results}

Respondent-driven sampling and characteristics of drug users

Recruitment started with 3 seeds: 2 men (1 low threshold service, 1 drug treatment center) and 1 woman (needle and syringe exchange program). The first chain recruited 117 UDs and was interrupted at the sixth wave. The second and third chains included 96 and 114 DUs, respectively, and were interrupted at the fourth wave. A total of 327 UDs were recruited in less than 2 months (February to March 2015). All the participants enrolled met the eligibility criteria. None of the 3 chains was interrupted for lack of new respondents. The mean number of DUs encountered by each respondent during the 15 days preceding the interview was 13.6 (95\% CI 11.6-15.5). Whatever item is chosen, a tendency toward in-group recruitment coexisted with considerable cross-group recruitment, but no barrier was observed between the groups as shown in Table 2 . Sex was the only characteristic demonstrating a negative homophily.

There was no statistical difference between the observed and weighted distribution of screening compared to injection (Table 3).

The mean age of our sample was 33.4 years $(95 \%$ CI 32.3-34.4). Mean age of the first drug experimentation was 15.02 years. Age of the first injection was 21 years (95\% CI 20.25-21.67). The mean number of drugs consumed during life was 8.66 (95\% CI 8.39-8.94). Socio-economic and drug-related characteristics of the participants are presented in Tables 4 and 5 .

\section{HCV screening}

Testing was not related to any socio-demographic characteristic such as sex, nationality, education, profession, social coverage, or housing. If friends were the second most frequent source (37\%) of health information after GPs (55\%) and before SDUs (27.5\%), their opinion did not influence HCV screening. No specific plan for the
Table 2 Homophily for sex, age, intravenous drug use, and HCV screening and serology

\begin{tabular}{ll}
\hline & Homophily \\
\hline Male & 0.093 \\
Female & -0.137 \\
Age & \\
$\quad<20$ & 0.198 \\
$20-29$ & 0.08 \\
$30-39$ & 0.114 \\
$40-49$ & 0.073 \\
$>50$ & 0.054 \\
Non-IVDU & 0.135 \\
IVDU & 0.118 \\
HCV screening+ HCV+ & 0.159 \\
HCV screening+ HCV- & 0.125 \\
HCV screening- & 0.154 \\
\hline
\end{tabular}

Homophily refers to the tendency to recruit in the same category with a metric between 1 (recruited and recruits in the same category) and -1 (recruited and recruits in a different category). Heckathorn suggests any value of homophily $\geq 0.3$ as intermediate homophily and any value $\leq-0.3$ as strong heterophily

future was associated with a previous test. There was no difference between DUs whose screening result was positive or negative.

HCV screening was associated with age (screening + 35.01 (95\% CI 33.95-36.08)/screening - 25.90 (95\% CI 23.33-28.46), $p<0.0001$ ) as well as duration of drug use (screening $+19.46(95 \%$ CI 18.43-20.49)/screening 11.37 (95\% CI 8.76-13.99), $p<0.0001$ ) and duration of injection (screening + 14.79 (95\% CI 13.14-16.44)/ screening -2.87 (95\% CI 1.60-4.14), $p<0.0001)$. The results of the other significant univariate tests and multivariate logistic regression models measuring the association of past year HCV testing and selected participant characteristics are presented in Table 6.

Multiples reasons (54/60) were given by respondents to explain the absence of screening. An absence of concern (85.2\%, 95\% CI 73.4-92.3) came first, followed by problems with blood access (22.2\%, 95\% CI 13.20-34.94). A fear of the result was mentioned by seven, and only three reported being unaware of voluntary testing sites. However, two thirds (61.3\%) accepted the theoretical proposal

Table 3 Observed and weighted distribution of the crosstabulation of screening by injection

\begin{tabular}{lll}
\hline Screening Injection & Yes, 95\% Cl & No, 95\% Cl \\
\hline $\begin{array}{lll}\text { Observed } \\
\text { Yes }\end{array}$ & $54.1,48.6-59.6$ & $7.6,4.8-105$ \\
No & $27.8,23-33$ & $10.4,7.3-14.2$ \\
Weighted & & \\
Yes & $50.8,42.5-58.8$ & $9.8,5.3-16.6$ \\
No & $27.8,20.8-33.1$ & $11.6,7-17.6$ \\
\hline
\end{tabular}


Table 4 Socio-professionnal characteristics of DUs (semiquantitative)

\begin{tabular}{|c|c|c|c|}
\hline & Observed nb & Percent & $95 \% \mathrm{Cl}$ \\
\hline \multicolumn{4}{|l|}{ Sex } \\
\hline Men & 243 & 74.3 & $69.6-79.0$ \\
\hline Women & 84 & 25.7 & $21.0-30.4$ \\
\hline \multicolumn{4}{|l|}{ Nationality } \\
\hline French & 275 & 84.1 & $80.1-88.1$ \\
\hline Foreigner & 52 & 15.9 & 11.9-19.9 \\
\hline \multicolumn{4}{|l|}{ Housing } \\
\hline Stable (owner or rent) & 141 & 43.1 & $37.9-48.5$ \\
\hline Parents & 38 & 11.6 & $8.6-15.6$ \\
\hline Friends & 22 & 6.7 & $4.5-10.0$ \\
\hline Shelter & 37 & 11.3 & $8.3-15.2$ \\
\hline Homeless, squat & 89 & 27.2 & $22.7-32.3$ \\
\hline \multicolumn{4}{|l|}{ Education } \\
\hline Primary & 10 & 3.1 & $1.7-5.5$ \\
\hline Secondary & 225 & 68.9 & $63.6-73.6$ \\
\hline Bachelor & 52 & 15.9 & $12.3-20.3$ \\
\hline Higher education & 40 & $12 \cdot 2$ & $8.7-15.8$ \\
\hline \multicolumn{4}{|l|}{ Home } \\
\hline Parents & 49 & 15 & $11.1-18.9$ \\
\hline Couple & 103 & 31.5 & $26.5-36.5$ \\
\hline Alone & 145 & 44.3 & $38.9-49.7$ \\
\hline Other & 30 & 9.1 & $6.0-12.2$ \\
\hline \multicolumn{4}{|l|}{ Profession } \\
\hline Studying & 10 & 3.1 & $1.0-5.0$ \\
\hline Permanent contract & 32 & 9.8 & $6.6-13.0$ \\
\hline Fixed term contract & 38 & 11.6 & $8.1-15.1$ \\
\hline Unemployed & 58 & 17.7 & $13.6-21.8$ \\
\hline Disability & 9 & 2.8 & $1.0-4.6$ \\
\hline Pension & 1 & 0.3 & $-0.3-0.9$ \\
\hline None & 179 & 54.7 & $49.3-60.1$ \\
\hline Active solidarity income & 150 & 45.9 & $40.6-51.3$ \\
\hline Prison & 162 & 49.5 & $44.08-54.92$ \\
\hline \multicolumn{4}{|l|}{ Sexual preference } \\
\hline Heterosexual & 295 & 90.2 & $85.5-93$ \\
\hline Bisexual & 26 & 8 & $5.5-11.4$ \\
\hline Homosexual & 6 & 1.8 & $0.84-3.9$ \\
\hline
\end{tabular}

for immediate screening, significantly lower (86\%) than those who had previously been screened (odds ratio 0.26 , 95\% CI 0.11-0.61, $p<0.001)$.

HBV (67.8\%, 95\% CI 67.79-72.63), HIV (86.8\%, 95\% CI 82.70-90.06), and HCV screening were very closely linked (HBV/HCV odds ratio 36.52, 95\% CI 14.9089.55; HIV/HCV odds ratio 23.53, 95\% CI 10.87-50.92) and were also linked to the same items (duration of drug use, access to treatment of drug use and, for HBV, police crackdown on injections).

\section{HCV contamination and treatment}

HCV prevalence (observed 22.8\%, weighted 21\%) was low. In a univariate analysis, HCV contamination was related to the risks related to drug use such as its duration ( $\mathrm{HCV}-17.9$ years, 95\% CI 16.8-22.9/HCV+ 24.9, 95\% CI 22.9-26.9), needle and syringe sharing (NSS), episodes of craving or police crackdown on injections, and history of care; once the level of risks and the duration of drug use were taken into account, care disappeared because they were strongly related to age and duration of drug use (Table 7). Sharing of works was not an additional risk of contamination. Access to sterile works was considered easy by $88.7 \%$ of current injectors who used syringe-dispensing machine, needle exchange program, bus, and pharmacy at the same time. It was not related to HCV screening or contamination. The high odds ratio associated with OCT is explained by this correlation.

A spontaneous clearance of $\mathrm{HCV}$ was reported in 11 cases (18\%, 95\% CI 10.4-29.5). Among those who had not spontaneously cleared HCV, 23 had been treated (46\%, 95\% CI 33-59.6, mean year 2005, range 19902015). Treatment had been completed in 21 cases, and 13 believed they had been cured. The decision to be treated was favored in 14 cases $(60.9 \%)$ by trust in the specialist or in the medical team. Among the 27 who had not been treated, the main reason was an absence of severity of hepatitis (59.2\%). The main cause of patients' refusal was side effects (33.3\%), followed by not feeling ill (25.9\%) and by problems with venous access (22.2\%). Alcohol was presented as preventing treatment for doctors ( 2 cases) as well as for patients (6 cases).

HBV (5.3\%, 95\% CI 2.99-9.26) and HIV (1.7\%, 95\% CI 0.75-4.03) were too low for a statistical analysis. Two of the $11 \mathrm{HBV}$-positive and 2 of the $5 \mathrm{HIV}$-positive DUs had never injected.

\section{Fear of contamination}

Fear of contamination was neither related to any previous risk of contamination (drug or condom use) nor to the duration of drug use or a previous HCV test. It was associated with planning a future HCV test that did not increase the rate of acceptance of an immediate screening proposal. Among the factors associated with fears of contamination, other fears (total number and fear of overdoses) came first (Table 8). For once, socio-demographic characteristics were concerned with a protection for DUs who worked with social security coverage and a stable housing and an increased prevalence among DUs with illegal incomes. 
Table 5 Characteristics related to drug use

\begin{tabular}{|c|c|c|c|}
\hline & Observed number & Percent & $95 \% \mathrm{Cl}$ \\
\hline \multicolumn{4}{|l|}{ History of injection } \\
\hline NSS+ & 114 & 34.9 & $29.9-40.2$ \\
\hline NSS- & 89 & 27.2 & $22.7-32.3$ \\
\hline None & 125 & 38.2 & $32.9-43.5$ \\
\hline \multicolumn{4}{|l|}{ Treatment for drug use } \\
\hline Never & 80 & 24.5 & $19.5-28.7$ \\
\hline Current & 202 & 61.8 & $56.5-67.1$ \\
\hline Given up & 45 & 13.9 & $10.1-17.5$ \\
\hline Drug treatment center & 59 & 28.7 & $23.32-33.08$ \\
\hline Low threshold services & 26 & 12.9 & $9.27-16.53$ \\
\hline Bus & 23 & 11.4 & $7.96-14.84$ \\
\hline GPs & 141 & 69.8 & $64.82-74.78$ \\
\hline \multicolumn{4}{|c|}{ Current opiate substitution therapy } \\
\hline Methadone & 85 & 26 & $21.3-31.1$ \\
\hline Buprenorphine & 65 & 19.9 & $15.7-24.6$ \\
\hline Morphine sulfate & 15 & 8.6 & $2.6-7.5$ \\
\hline \multicolumn{4}{|l|}{ Fears } \\
\hline Prison & 161 & 49.2 & $43.9-54.6$ \\
\hline Not stopping drug use & 146 & 44.6 & $39.4-50.0$ \\
\hline Contamination & 143 & 43.7 & $38.5-49.2$ \\
\hline Staying alone & 138 & 42.2 & $37.0-47.6$ \\
\hline Losing one's friends & 117 & 35.8 & $30.8-41.1$ \\
\hline Overdose & 91 & 27.8 & $23.3-32.9$ \\
\hline Not paying one's debts & 74 & 22.6 & $18.4-27.5$ \\
\hline Stopping drug use & 69 & 21.1 & $17.0-25.9$ \\
\hline Losing custody of children & 44 & 13.5 & $15.6-24.2$ \\
\hline Losing contact with friends & 34 & 10.4 & $7.54-14.2$ \\
\hline Losing one's job & 32 & 9.8 & $7.0-13.5$ \\
\hline \multicolumn{4}{|l|}{ Sources of health information } \\
\hline GP & 180 & 55 & $49.6-60.4$ \\
\hline Friends & 121 & 37 & $32.0-42.4$ \\
\hline SDU & 90 & 27.5 & $23.0-32.6$ \\
\hline Internet (computer) & 89 & 27.2 & $22.7-32.3$ \\
\hline Television & 86 & 26.3 & $21.8-31.3$ \\
\hline Booklet & 85 & 26 & $21.5-31.0$ \\
\hline Stand & 50 & 15.3 & 11.8-19.6 \\
\hline Family & 46 & 14.1 & $10.7-18.3$ \\
\hline Press (free) & 42 & 12.8 & $9.6-16.9$ \\
\hline Internet (smartphone) & 26 & 8 & $5.5-11.4$ \\
\hline Social networks & 25 & 7.6 & $5.2-11.0$ \\
\hline Press (paid for) & 23 & 7 & $1.7-10.3$ \\
\hline \multicolumn{4}{|l|}{ HCV screening } \\
\hline Adequate & 241 & 73.7 & $68.7-78.2$ \\
\hline Inadequate & 26 & 7.95 & $5.5-11.4$ \\
\hline
\end{tabular}

Table 5 Characteristics related to drug use (Continued)

\begin{tabular}{llll}
\hline & Observed number & Percent & $95 \% \mathrm{Cl}$ \\
\hline Absent & 60 & 18.3 & $15.5-22.9$ \\
Positive & 61 & $22.8^{\mathrm{a}}$ & $18.2-28.3$ \\
Negative & 206 & $77.2^{\mathrm{a}}$ & $71.8-81.8$ \\
Unknown & 60 & $18.4^{\mathrm{b}}$ & $14.5-22.9$ \\
\hline
\end{tabular}

NSS needles and syringes sharing, GP source GP as source of health information, none no injection, $S D U$ service for drug users, SDU source service for drug users as source of health information, Source of information source of health information, $\mathrm{HCV}$ screening adequate less than 1 year or absence of injection since the last test, Inadequate current injector with more than 1 year since the lest test, absent no test

aprevalence among screened DUs

${ }^{b}$ Prevalence among the 327 DUs

\section{Knowledge of the efficacy of HCV treatments}

An accurate knowledge of the efficacy of $\mathrm{HCV}$ treatments (37.9\%; OR CI 32.6-43.4) was higher among seropositive DUs (+72.1/others $30.2 \%$; odds ratio 95\% CI $3.23-11.14 ; p<0.0001)$. The knowledge of the efficacy of $\mathrm{HBV}$ and $\mathrm{HCV}$ treatments and of $\mathrm{HBV}$ vaccination were higher among HCV-positive respondents than HCV-negative ones. Among seronegative cases, GPs were the only source of accurate knowledge (Table 9).

\section{Plans for the future}

Looking for a job (62.4\%; 95\% CI 56.9-67.7) was the main concern among the 28 possible projects. It was followed by taking care of one's teeth $(58.7 \%$; $95 \% \mathrm{CI}$ $53.2-64.1)$, returning to activity $(42.8 \%$; $95 \%$ CI $37.4-$ 48.4), reducing drug use (40.7\%; 95\% CI 35.3-46.2), and making a health check (40.4\%; 95\% CI 35-45.9). Making a health check was unrelated to age, NSS, HCV screening, and serology results as well as seeking health information from GPs or DUs' services. A more specific project of HCV screening (11.9\%; 95\% CI 8.6-15.9) and engaging in one's hepatitis treatment $(5.8 \%$; $95 \% \mathrm{CI}$ $3.5-8.9)$ ranked 25th and 28th, respectively. The only item significantly associated with plans of a future screening for hepatitis was the fear of contamination.

\section{Discussion}

This study, the first to use the RDS method to recruit DUs in France, recruited a population younger and with lower HCV prevalence than those of other French studies that included IV- and non-IVDUs [32-34] because of the inclusion of users left outside treatments for drug use (38.2\%) who were previously not studied. Compared to the previous snowball sampling, RDS was significantly superior to recruit this hidden population (OR 115.4, 95\% CI 28.12-474.81) The interviews confirmed Heckathorn's initial hypothesis that money was not the only reason for participating in the investigation [35]. Respondents were eager to have the opportunity, for once, to tell the story of their lives. All these 
Table 6 Factors significantly associated with an HCV test in univariate tests and binary logistic regression (forward stepwise selection)

\begin{tabular}{|c|c|c|c|c|c|c|}
\hline \multirow[t]{2}{*}{ HCV screening } & \multirow[t]{2}{*}{$\%$ screening } & \multirow[t]{2}{*}{$\%$ total } & \multirow[t]{2}{*}{ Sig } & \multirow[t]{2}{*}{ Odds ratio } & \multicolumn{2}{|l|}{ 95\% Cl odds ratio } \\
\hline & & & & & Lower & Upper \\
\hline Injection & & & 0.002 & 0.378 & 0.213 & 0.672 \\
\hline Yes & 87.2 & 62.1 & & & & \\
\hline No & 73.4 & 37.9 & & & & \\
\hline NSS & & & $<0.001$ & 5.363 & 2.476 & 14.358 \\
\hline Yes & 94.7 & 34.9 & & & & \\
\hline No & 75.1 & 65.1 & & & & \\
\hline Craving & & & $<0.0001$ & 4.392 & 1.819 & 10.606 \\
\hline Yes & 93.7 & 29.1 & & & & \\
\hline No & 77.2 & 70.9 & & & & \\
\hline Police & & & $<0.0001$ & 9.344 & 3.257 & 26.807 \\
\hline Yes & 96.3 & 38.3 & & & & \\
\hline No & 73.6 & 61.7 & & & & \\
\hline GP source & & & $<0.0001$ & 5.231 & 2.735 & 10.005 \\
\hline Yes & 92.2 & 55.0 & & & & \\
\hline No & 69.4 & 45.0 & & & & \\
\hline SDU source & & & 0.008 & 3.810 & 1.276 & 6.188 \\
\hline Yes & 91.1 & 27.5 & & & & \\
\hline No & 78.5 & 72.5 & & & & \\
\hline SDU or GP & & & $<0.0001$ & 6.772 & 3.566 & 12.861 \\
\hline Yes & 92.6 & 61.8 & & & & \\
\hline No & 65.7 & 38.2 & & & & \\
\hline Prison & & & $<0.0001$ & 7.833 & 3.555 & 17.261 \\
\hline Yes & 94.7 & 49.5 & & & & \\
\hline No & 69.7 & 50.5 & & & & \\
\hline \multirow[t]{2}{*}{ Logistic regression } & B & Wald & Sig & Odds ratio & 95\% Cl odds ratio & \\
\hline & & & & & Lower & Upper \\
\hline \multicolumn{7}{|l|}{ All: 327 cases } \\
\hline Age & 0.099 & 12.764 & $<0.0001$ & 1.100 & 1.044 & 1.159 \\
\hline Police & -1.338 & 7.060 & 0.008 & 0.262 & 0.098 & 0.704 \\
\hline OST & -1.750 & 9.311 & 0.002 & 0.174 & 0.057 & 0.535 \\
\hline GPsource & -1.014 & 6.347 & 0.012 & 0.362 & 0.165 & 0.798 \\
\hline Prison & -1.073 & 4.748 & 0.029 & 0.342 & 0.130 & 0.898 \\
\hline Constant & 1.593 & 2.051 & 0.152 & 4.921 & & \\
\hline
\end{tabular}

Hosmer and Lemeshow test, 0.101; CC-screening+, 94.8\%; screening-, 44.8\%; all, 85.8\%

Logistic regression odds ratios_-police: absent/present, injection: NSS- and no injection/NSS+, OST: absent/present, GP source: absent/present

Injection history of injection, NSS needle and syringe sharing, Craving NSS at a time of craving, Police police crackdown on injections, GP source GP as source of health information, SDU service for drug users, GP medical practice of a general practitioner, OST opiate substitution therapy, prison having served a prison sentence, CC correctly classified

DUs belonged to a diverse community whose only common feature was drug use with no barriers between injectors and non-injectors. $\mathrm{HCV}$ prevalence was low (overall 22.8\%, IVDUs 33.3\%), and HIV and HBV prevalence (5.3 and $1.7 \%$ ) were too low to allow any statistical analysis.
Our results have points of divergence and concordance with respect to previous studies on the same subject.

1. The main difference concerns the socio-economic dimensions almost always present outside France $[17,36]$. In our study, it had a very little influence 
Table 7 Factors significantly associated with an HCV positive ( $\mathrm{HCV}+$ ) test in univariate tests and binary logistic regression (forward stepwise selection)

\begin{tabular}{|c|c|c|c|c|c|c|}
\hline \multirow[t]{2}{*}{$\mathrm{HCV}+$} & \multirow[t]{2}{*}{$\% \mathrm{HCV}+$} & \multirow[t]{2}{*}{$\%$ total } & \multirow[t]{2}{*}{ Sig } & \multirow[t]{2}{*}{ Odds ratio } & \multicolumn{2}{|c|}{ 95\% Cl odds ratio } \\
\hline & & & & & Lower & Upper \\
\hline Injection & & & $<0.0001$ & 22.250 & 5.294 & 93.518 \\
\hline Yes & 33.3 & 66.3 & & & & \\
\hline No & 3.3 & 33.7 & & & & \\
\hline NSS & & & $<0.0001$ & 15.579 & 7.205 & 33.688 \\
\hline Yes & 48.1 & 40.3 & & & & \\
\hline No & 5.6 & 59.7 & & & & \\
\hline Craving & & & $<0.0001$ & 9.26 & 4.83 & $4.83-17.74$ \\
\hline Yes & 49.4 & 33.2 & & & & \\
\hline No & 9.5 & 66.6 & & & & \\
\hline Police & & & $<0.0001$ & 3.519 & 1.858 & 6.663 \\
\hline Yes & 36.5 & 44.6 & & & & \\
\hline No & 14.1 & 55.2 & & & & \\
\hline SDU or GP & & & $<0.0001$ & 0.191 & 0.100 & 0.366 \\
\hline Yes & 29.9 & 69.8 & & & & \\
\hline No & 6.2 & 30.2 & & & & \\
\hline OST & & & $<0.0001$ & 4.959 & 2.444 & 10.060 \\
\hline Yes & 29.8 & 67.8 & & & & \\
\hline No & 8.1 & 32.2 & & & & \\
\hline SDU & & & $<0.0001$ & 3.420 & 1.814 & 6.450 \\
\hline Yes & 42.1 & 21.3 & & & & \\
\hline No & 17.5 & 78.7 & & & & \\
\hline Prison & & & 0.009 & 2.279 & 1.212 & 4.274 \\
\hline Yes & 29.9 & 57.4 & & & & \\
\hline No & 15.7 & 42.6 & & & & \\
\hline \multirow[t]{2}{*}{ Logistic regression } & $B$ & Wald & Sig & Odds ratio & $95 \% \mathrm{Cl}$ & \\
\hline & & & & & Lower & Upper \\
\hline NSS & 0.108 & 17.440 & $<0.0001$ & 1.115 & 1.080 & 1.174 \\
\hline Drug use & -2.623 & 33.403 & $<0.0001$ & 0.73 & 0.30 & 0.177 \\
\hline Police & -1.190 & 8.310 & 0.004 & 0.323 & 0.150 & 0.697 \\
\hline Constant & -1.939 & 8.310 & 0.002 & & & \\
\hline
\end{tabular}

Hosmer and Lemeshow test, 0.991; CC-HCV+, 51.8\%; HCV-,90.3\%; all, 81\%

Injection history of injection, NSS needle and syringe sharing, Craving NSS at a time of craving, Police police crackdown on injections, SDU service for drug users, GP medical practice of a general practitioner, OST opiate substitution therapy, Drug treatment center attending SDU or GP, Drug use duration of drug use, CC correctly classified

Logistic regression odds ratios: NSS absent/present, police: absent/present, injection: NSS- and no injection/NSS+, craving: injection at a time of craving-/injection at a time of craving+ (267 cases: exclusion of HCV+ DUs)

on HCV screening, in spite of $17.7 \%$ of unemployed, a high rate of respondents outside of the labor force (54.7\%), or without a stable housing (45.2\%). These differences may be related to the French welfare model, with a universal and free access to OST, and to the rich and diversified supply of free harm reduction programs and healthcare services in Strasbourg.
2. With an overall reported screening rate of $82 \%$ and $46 \%$ of those found PCR-positive treated, one could consider these results as optimal, much higher than usually reported [7, 13, 37]. However, once analyzed following subpopulations of DUs, it did not appear so positive. First, if $92.6 \%$ of DUs attending SDUs had been screened, this rate fell to $75.6 \%$ for those who had dropped out and 58.8\% for those who had 
Table 8 Factors significantly associated with fear of contamination in univariate tests and binary logistic regression

\begin{tabular}{|c|c|c|c|c|c|c|}
\hline \multirow[t]{2}{*}{ Fear of contamination } & \multirow[t]{2}{*}{$\%$ fear } & \multirow[t]{2}{*}{$\%$ total } & \multirow[t]{2}{*}{ Sig } & \multirow[t]{2}{*}{ Odds ratio } & \multicolumn{2}{|c|}{ 95\% Cl odds ratio } \\
\hline & & & & & Lower & Upper \\
\hline \multicolumn{7}{|l|}{ Univariate } \\
\hline Health & & & 0.009 & 1.937 & 1.179 & 3.182 \\
\hline Yes & 52.7 & 41.4 & & & & \\
\hline No & 36.5 & 56.6 & & & & \\
\hline Hepatitis & & & $<0.0001$ & 5.419 & 1.947 & 15.080 \\
\hline Yes & 78.3 & 8.6 & & & & \\
\hline No & 39.9 & 91.4 & & & & \\
\hline Overdose & & & $<0.0001$ & 5.292 & 2.936 & 9.537 \\
\hline Yes & 71.9 & 27.8 & & & & \\
\hline No & 32.2 & 72.7 & & & & \\
\hline Illicit* & & & 0.004 & & & \\
\hline Legal & 35.4 & 55.3 & & & & \\
\hline Moonlighting & 43.9 & 18.9 & NS & 1.42 & 0.73 & 2.76 \\
\hline Illegal & 59.2 & 26.7 & $<0.001$ & 2.65 & 1.18 & 4.74 \\
\hline Social inclusion & & & $<0.0001$ & 0.11 & 0.03 & 0.375 \\
\hline Yes & 9.1 & 12.4 & & & & \\
\hline No & 48.1 & 87.6 & & & & \\
\hline \multirow[t]{2}{*}{ Logistic regression } & B & Wald & Sig & Odds ratio & $95 \% \mathrm{Cl}$ & \\
\hline & & & & & Lower & Upper \\
\hline Fears $\mathrm{Nb}$ & 0.580 & 27.333 & $<0.0001$ & 1.751 & 1.419 & 2.160 \\
\hline Social inclusion & -1.851 & 6.347 & 0.012 & 0.157 & 0.037 & 0.863 \\
\hline Hepatitis & -1.766 & 7.881 & 0.005 & 0.171 & 0.050 & 0.587 \\
\hline overdose & -0.939 & 6.047 & 0.014 & 0.314 & 0.185 & 0.827 \\
\hline \multicolumn{7}{|l|}{ Illicit* } \\
\hline Legal & -0.973 & 6.673 & 0.010 & 0.378 & 0.181 & 0.791 \\
\hline Moonlighting & -1.120 & 4.745 & 0.029 & 0.391 & 0.185 & 0.827 \\
\hline Constant & 2.933 & 6.552 & 0.010 & 18.790 & & \\
\hline
\end{tabular}

Hosmer and Lemeshow test, 0.777; CC-Fear+, 71.9\%; Fear-, 85.1\%; all, 79.9\%

Logistic regression odds ratios: social inclusion (forward stepwise selection) (266 cases: exclusion of HCV+ DUs)

Fears $\mathrm{Nb}$ total number of fears, Health plan for a health check, Hepatitis plan for HCV screening, Overdose fear of overdoses, Illegal drug trafficking or theft, Social inclusion having a job, housing, social security

never attended a SDU. Moreover, $7.9 \%$ of the screening had been performed more than 1 year before our study. Overall, an optimal screening would have missed $33.9 \%$ (95\% CI 28.8-39.4) of the recruited DUs. If hepatitis $C$ had been a major concern of the DUs, the screenings would have been the result of active and rational approaches, correlated with past risk-taking, sources of concern about possible contaminations, and a search for information about the effectiveness of treatments. Our data, at odds with this presumption, favor the hypothesis of a moderate interest of the UDs for the hepatitis $\mathrm{C}$ inducing little active screening.

(a) First, $85.2 \%$ of the DUs who had not been screened explained this absence by their lack of concern about HCV hepatitis. Then, we did not find any correlation between the presence of appropriate screening and previous risk-taking. A belief in the efficacy of HCV treatments was present in only $30.1 \%$ of those DUs who had not been screened positive, and $42.8 \%$ of those who had been screened DUs had no opinion concerning the efficacy of HCV treatments. This limited knowledge is consistent with the literature [17]. At last, the main plans for the future had a concrete and immediate dimension such as work, teeth, and housing. Hepatitis ranked almost last when DUs did not know they were infected. Our study provides elements to understand this lack of correlation: 
Table 9 Factors significantly associated with the knowledge of the efficacy of HCV treatment in univariate tests and binary logistic regression (forward stepwise selection) among HCV negative respondents

\begin{tabular}{|c|c|c|c|c|c|c|}
\hline \multirow[t]{2}{*}{$\mathrm{HCV}$ treatment } & \multirow[t]{2}{*}{$\%$ treatment } & \multirow[t]{2}{*}{$\%$ total } & \multirow[t]{2}{*}{ Sig } & \multirow[t]{2}{*}{ Odds ratio } & \multicolumn{2}{|l|}{ 95\% Cl odds ratio } \\
\hline & & & & & Lower & Upper \\
\hline \multicolumn{7}{|l|}{ Univariate } \\
\hline GP source & & & 0.001 & 2.545 & 1.473 & 4.395 \\
\hline Yes & 39.6 & 50.4 & & & & \\
\hline No & 20.5 & 49.6 & & & & \\
\hline $\mathrm{GP}$ & & & $<0.0001$ & 2.919 & 1.700 & 5.009 \\
\hline Yes & 43.5 & 40.6 & & & & \\
\hline No & 20.9 & 59.4 & & & & \\
\hline HBV treatment & & & $<0.0001$ & 3.770 & 2.176 & 6.531 \\
\hline Yes & 48.0 & 36.8 & & & & \\
\hline No & 19.6 & 63.2 & & & & \\
\hline HBV vaccine & 49.6 & & $<0.0001$ & 3.377 & 1.928 & 5.915 \\
\hline Yes & 42.4 & 49.6 & & & & \\
\hline No & 17.8 & 50.4 & & & & \\
\hline \multirow[t]{2}{*}{ Logistic regression } & B & Wald & sig & Odds ratio & 95\% $\mathrm{Cl}$ odds ratio & \\
\hline & & & & & Lower & Upper \\
\hline GP & -0.853 & 8.499 & 0.004 & 0.426 & 0.240 & 0.758 \\
\hline GP source & -0.648 & 4.720 & 0.030 & 0.523 & 0.291 & 0.939 \\
\hline Constant & -0.088 & 0.176 & 0.675 & 0.916 & & \\
\hline
\end{tabular}

Hosmer and Lemeshow test, 0.104; CC-Knowledge+, 0\%; Knowledge-, 100\%; all, 69.9\%

Logistic regression odds ratios: GP absent/present, GP source: absent/present (266 cases: exclusion of HCV+ DUs)

HCV treatment accurate knowledge of the efficacy of HCV treatment, GP source general practitioner as source of health information, GP medical practice of a general practitioner, HBV treatment knowledge of the efficacy of HBV treatment, HBV vaccine knowledge of the efficacy of HBV vaccine

- We have found a positive impact of a police crackdown on injections on HCV screening, already known to increase contaminations $[38,39]$. When one believes, rightly or wrongly, that his usual drug use does not present a specific risk, police interventions, modifying these routines, reveal a risk that has not been internalized and lead to an active hitherto nonexistent decision of screening, even in the absence of a current contact with healthcare providers. As in any educational approach, one is faced with the difficulty of trivializing (internalization of imposed norms) a non-trivial (human) subject [40]. "Being not concerned," given as the main reason for not having performed $\mathrm{HCV}$ tests, may be considered as reflecting a denial, allowing the acceptance of what has become an unconscious contradiction and repressing any conscious thought of an inevitable death [41]. This blindness is favored by the absence of clear messages hierarchizing the risks [16, 17]; if NSS and sharing of works are placed at the same level as sexual intercourse without a condom, sharing sniffing or smoking paraphernalia, toothbrushes, and razors as well as tattoos, is it possible to avoid contamination?

- Fear of contamination that affected almost half of the respondents was independent of the use of drugs (history of injection or NSS), of sexual practices (use of condoms), or of a previous screening. It had only one "medical" association: the project of a future $\mathrm{HCV}$ screening. But this plan did not result in a better acceptance of an immediate screening. If drug use was one of its key determinants (strong relationship between fear of contamination and overdoses), other factors came into play such as the total number of fears. It was reduced by a very good social integration and increased by illegal activities. This can be seen as inexplicable with respect to the actual risks of contamination, but from DUs' point of view, it can be considered as the result of individual "ecological" imaginary constructs reflecting the diversity of their priorities among which the 
understanding of the message associated with the risks of contamination is part of a global representation of a dangerous lifestyle.

(b) If hepatitis B and C screenings, like hepatitis $B$ vaccinations, were not associated with any risk previously taken, they were correlated with attendance to SDUs and, in particular, with the relationship (sources of information,

prescription) of DUs with GPs. This finding is in accordance with a passively accepted screening already observed by Jordan et al. [19]. The significant role of the screening proposed systematically at the entry into prison as well as the acceptance of an immediate test by all seronegative DUs whose tests were too old illustrates this passive position and the need to remind professionals of the value of repeating tests when risk-taking persists even if DUs do not require it. In our experience, this passive acceptance, like that of liver biopsies or hepatitis treatments, works often as a reciprocal tradeofflike exchange where the test (biopsy, treatment) operates, besides and in addition to trust, as a symbolic payment (counter-gift) for the initial gift of the care, provided that its payment is not considered to have been paid in full [42]. Acceptance of screening was favored by the capacity of the professional to provide accurate information on hepatitis B and C and on their treatments as well as on vaccinations as underlined by the centrality of GPs among all health care providers. Observation is already made by Walley and al. [21]. This capacity to explain is mandatory to make the existence of a life-threatening disease credible whose only symptom is most often a non-specific fatigue and of its possible cure [43, 44]. It is the reason for the success of integrated care interventions [45-47] practiced in Strasbourg with an overall rate of treatment higher than usually reported $[48,49]$.

Ten years later, our assumptions are in line with Treloar and Rhodes's and expand their conclusion [16]. Justifying our inclusion of non-injectors, we show, for the first time, that there is no difference in this life experience between injectors and non-injectors, confirming the difficulty encountered in understanding and integrating prevention messages which hinder the access to screening and treatment [18].

The low prevalence of HCV and HIV infections observed confirms the trend toward a decline of both incidence already observed in France [32-34, 50] and in other countries [51]. Since we have shown, after others, that risk-taking is not associated with a clear understanding of viral contaminations, this trend cannot be attributed to changes in behavior following a rational risk analysis. It is more likely that the lasting impact induced by the AIDS epidemic on behaviors followed a group mimicry which can be likened to a heuristic strategy [52] known to spread among social networks like epidemics [53-56]. Its impact was favored by access to syringes (in France as early as 1987), OST (in France universal and free access in 1996), and other harm reduction programs where DUs meet care providers. In spite of these positive findings, we can conclude that the control of the HCV epidemic will not come by improving the delivery of targeted prevention message. They will never have the efficacy of the early AIDS messages that had all the characteristics required of a message to perform [57]; it was simple (HIV infection led to death), unexpected (people paid attention), concrete (it was understood and remembered), credible (people agreed and believed), and emotional (people feared) and led people to act (a credible story was told with a simple solution: condoms and sterile works). In addition, access to sterile works was considered satisfying by more than four fifth of injectors and will be difficult to improve significantly. Last, limited access to DAA to the most advanced liver diseases, besides its inefficiency to control the epidemic, maintains the confusion of the messages. Treatment of what is presented as a life-threatening disease would be eligible to only one third of infected UDs if their contamination is old enough to result in a severe liver disease, and, even in that case, the continued use of drugs or alcohol could be sufficient to deny it $[13,58,59]$.

\section{Conclusion}

As was proposed for HIV and $\operatorname{HCV}[60,61]$, a universal access to DAA is the best approach to impact significantly the course of the epidemic, provided that most infected DUs are screened and treated for a period short enough to prevent reinfection. We have demonstrated that in a large French city where services are exceptionally developed and free, this objective may not be achieved due to a too small active investment of the DUs. We have to move from a predominant selective screening of DUs attending SDUs to a screening of its entire population. Considering the results of our RDS investigation which is a kind of conditional cash transfer, we believe that this approach could be a solution. With resources much lower than those needed to fund outreach teams, it would be able to recruit UDs who are not interested in SDUs' offers. The nudge $[62,63]$ would be the association, that has been effective, of a cash incentive, participation in public health action, and the opportunity to tell one's story. Providing respondents with an $\mathrm{HCV}$ education opportunity, that was not supplied by our study [21], should, with the possible access to an effective treatment through a shared 
decision [64], improve the rate of acceptance of $\mathrm{HCV}$ screenings and treatments. This access, possible in France, removes any concern about the ethical nature of this incentive. This approach would have the additional benefit of improving SDUs through a better knowledge of drug use and DUs' expectations in the targeted area, and the induced behavioral changes could spread among the DU community through peers' example [53-56, 65]. Conditional cash transfer has already been successfully used for diverse health programs [66-69]. This hypothesis will need an actual study to be verified.

\section{Abbreviations}

DAA: Direct-acting antiviral; DU: Drug user; NSS: Needle and syringe exchange; OST: Opioid substitution treatment; RDS: Respondent-driven sampling; SDU: Service for DUs; Source: Source of health information

\section{Acknowledgements}

We thank Ithaque's team and the DUs who helped us to build the questionnaire.

\section{Funding}

This research was funded by the "Fonds de dotation Gilead Sciences." The grand was awarded by a committee independent of Gilead Sciences. Role of the funding source, GILEAD, had no role in the study design; the collection, analysis, or interpretation of the data; or in the writing or submission of the manuscript.

\section{Availability of data and materials}

The datasets used and/or analyzed during the current study are available from the corresponding author on reasonable request.

\section{Authors' contributions}

PC and FDN conceived the study and supervised all aspects of its conduct. PC conducted the statistical analysis. PC and FDN wrote the first and subsequent manuscript drafts. Both authors read and approved the final manuscript.

\section{Authors' information}

PC was involved in the treatment of hepatitis B, C, and D since the end of the 1970s and of HIV infections since the beginning of the AIDS epidemic. He was the co-founder of a healthcare network for DUs in Lyon in 1993 and participated in the creation of the National Healthcare Network in 1997 of which he has been President until 2003.

\section{Ethics approval and consent to participate}

This study was approved by the French Commission Nationale de I'Informatique et des Libertés (CNIL). The database was anonymized. Apart from the three initial seeds to whom the study was presented by FD, all the other respondents were recruited by peers who explained the setting of the study. They decided voluntarily to be included in the study even before getting in touch with FD. All recruited DUs gave an informed consent to participate and to publication.

\section{Competing interests}

The authors declare that they have no competing interests (FDs' salary was paid by the grant)

\section{Publisher's Note}

Springer Nature remains neutral with regard to jurisdictional claims in published maps and institutional affiliations.

\section{Author details}

${ }^{1}$ UHSI de Lyon, Centre Hospitalier Lyon SUD, Hospices Civils de LYON, Chemin du Grand Revoyet, 69495 Pierre-Bénite, France. ${ }^{2}$ Association ITHAQUE, 12 rue Kuhn, 67000 Strasbourg, France.
Received: 9 December 2017 Accepted: 6 March 2018

Published online: 12 December 2018

\section{References}

1. Nelson PK, Mathers BM, Cowie B, Hagan H, Des Jarlais D, Horyniak D, Degenhardt L. Global epidemiology of hepatitis B and hepatitis $C$ in people who inject drugs: results of systematic reviews. Lancet. 2011;378(9791):57183. https://doi.org/10.1016/S0140-6736(11)61097-0.

2. La Torre G, Mannocci A, Saulle R, Colamesta V, Meggiolaro A, Mipatrini D, Sinopoli A. Economic evaluation of HBV vaccination: a systematic review of recent publications (2000-2013). Hum Vaccin Immunother. 2016:12: 2299-311.

3. Viganò $M$, Invernizzi $F$, Lampertico P. Optimal therapy of chronic hepatitis B: how do I treat my HBeAg-negative patients? Liver Int. 2015:35(Suppl 1):107-13.

4. Lin $\mathrm{CL}$, Yang HC, Kao JH. Hepatitis B virus: new therapeutic perspectives. Liver Int. 2016:36 Suppl 1:85-92.

5. Poorolajal J, Hooshmand E, Mahjub H, Esmailnasab N, Jenabi E. Survival rate of AIDS disease and mortality in HIV-infected patients: a meta-analysis. Public Health. 2016;139:3-12. https://doi.org/10.1016/j.puhe.2016.05.004

6. Burki T. Elimination on the agenda for hepatitis C. Lancet Infect Dis. 2014;14:452-3.

7. Lazarus JV, Sperle I, Maticic M, Wiessing L. A systematic review of hepatitis C virus treatment uptake among people who inject drugs in the European region. BMC Infect Dis. 2014;14(Suppl 6):S16. https://doi.org/10.1186/14712334-14-S6-S16.

8. Bonner JE1, Barritt AS 4th, Fried MW, Evon DM. Time to rethink antiviral treatment for hepatitis $C$ in patients with coexisting mental health/ substance abuse issues. Dig Dis Sci 2012; 57(6):1469-1474. https://doi.org/ 10.1007/s10620-012-2141-4.

9. Coward S, Leggett L, Kaplan GG, Clement F. Cost-effectiveness of screening for hepatitis C virus: a systematic review of economic evaluations. BMJ Open. 2016:6(9):e011821. https://doi.org/10.1136/bmjopen-2016-011821.

10. Sutton AJ, Hope VD, Mathei C, Mravcik V, Sebakova H, Vallejo F, et al. A comparison between the force of infection estimates for blood-borne viruses in injecting drug user populations across the European Union: a modelling study. Viral Hepat. 2008;15:809-16.

11. Philippe Chossegros (October 19th 2012). HCV and Drug Use - What Can Be Learned from the Failure to Control This Epidemic?, Addictions, David Belin, IntechOpen. https://doi.org/10.5772/50836. Available from: https:// www.intechopen.com/books/addictions-from-pathophysiology-totreatment/hcv-and-drug-use-what-can-be-learned-from-the-failure-tocontrol-this-epidemic.

12. Rafiq SM, Banik GR1, Khan S, Rashid H, Khandaker G. Current Burden of Hepatitis C Virus Infection Among Injecting Drug Users: A Mini Systematic Review of Prevalence Studies. Infect Disord Drug Targets. 2014;14:93-100.

13. Wiessing L, Ferri M, Grady B, Kantzanou M, Sperle I, Cullen KJ, EMCDDA DRID group, Hatzakis A, Prins M, Vickerman P, Lazarus JV, Hope VD, Matheî C. Hepatitis $C$ virus infection epidemiology among people who inject drugs in Europe: a systematic review of data for scaling up treatment and prevention. PLoS One. 2014;9(7):e103345. https://doi.org/10.1371/journal. pone.0103345.

14. Cooke GS, Hallett TB. HCV and HIV: shared challenges, shared solutions. Lancet Infect Dis. 2016;16:755-6.

15. Dhumeau D. Prise en charge des hépatites recommandation hépatites B et C. ANRS-AFEF 2014 Available at : http://www.sante.gouv.fr/IMG/pdf/ Rapport_Prise_en_charge_Hepatites_2014.pdf. Accessed Jan 2018.

16. C T, Rhodes T. The lived experience of hepatitis $C$ and its treatment among injecting drug users: qualitative synthesis. Qual Health Res. 2009;19:1321-34. https://doi.org/10.1177/1049732309341656.

17. Strauss SM, Astone-Twerell J, Munoz-Plaza CE, Des Jarlais DC, Gwadz M, Hagan $\mathrm{H}$, Osborne A, Rosenblum A. Drug treatment program patients' hepatitis $\mathrm{C}$ virus ( $\mathrm{HCV}$ ) education needs and their use of available HCV education services. BMC Health Serv Res. 2007;8(7):39.

18. J B, Brennan MB, Hull SJ, Stokes S, Fangman JJ, Westergaard RP. Barriers and facilitators of hepatitis $C$ screening among people who inject drugs: a multicity, mixed-methods study. Harm Reduct J. 2014;11:1. https://doi.org/10. 1186/1477-7517-11-1.

19. Jordan AE, Masson CL, Mateu-Gelabert P, McKnight C, Pepper N, Bouche K, Guzman L, Kletter E, Seewald RM, Des-Jarlais DC, Sorensen JL, Perlman DC. Perceptions of drug users regarding hepatitis $C$ screening and care: a 
qualitative study. Harm Reduct J. 2013;10:10. https://doi.org/10.1186/14777517-10-10.

20. Swan D, Long J, Carr O, Flanagan J, Irish H, Keating S, Keaveney M, Lambert J, McCormick PA, McKiernan S, Moloney J, Perry N, Cullen W. Barriers to and facilitators of hepatitis $C$ testing, management, and treatment among current and former injecting drug users: a qualitative exploration. AIDS Patient Care STDs. 2010;24:753-62. https://doi.org/10.1089/apc.2010.0142

21. Walley AY, White MC, Kushel MB, Song YS, Tulsky JP. Knowledge of and interest in hepatitis $C$ treatment at a methadone clinic. J Subst Abus Treat. 2005:28:181-7.

22. White RG, Hakim AJ, Salganik MJ, Spiller MW, Johnston LG, Kerr L, Kendall C, Drake A, Wilson D, Orroth K, Egger M, Hladik W. Strengthening the Reporting of Observational Studies in Epidemiology for respondent-driven sampling studies: "STROBE-RDS" statement. Clin Epidemiol. 2015;68(12): 1463-71. https://doi.org/10.1016/j.jclinepi.2015.04.002.

23. Sionean C, Le BC HK, Oster AM, Wejnert C, Hess KL, Paz-Bailey G, NHBS study group, Centers for Disease Control and Prevention (CDC). HIV Risk, prevention, and testing behaviors among heterosexuals at increased risk for HIV infection--National HIV Behavioral Surveillance System, 21 U.S. cities, 2010. MMWR Surveill Summ. 2014;63:1-39.

24. Centers for Disease Control and Prevention. HIV infection risk, prevention, and testing behaviors among men who have sex with men-National HIV Behavioral Surveillance, 20 U.S. cities, 2014. HIV Surveillance Special Report 15. Available at: http://www.cdc.gov/hiv/library/reports/surveillance/\#panel2 Published January 2016. Accessed Nov 2018.

25. Methods and definitions. European Monitoring Centre for Drugs and Drug Addiction http://www.emcdda.europa.eu/stats07/PDU/methods (accessed in jaanuary 2018).

26. Scheinmann R, Hagan H, Lelutiu-Weinberger C, Stern R, Des Jarlais DC, Flom PL, Strauss S. Non-injection drug use and Hepatitis C Virus: a systematic review. Drug Alcohol Depend. 2007;15(89):1-12.

27. Janssen E, Bastie A. Usage problématique de drogues en France: les prévalences en 2011.: OFDT Focus. 2103. /Consommation et conséquences. Available at: http://www.ofdt.fr/BDD/publications/docs/epfxejt9.pdf. Accessed Nov 2018.

28. Blackender $L$, Prestidge J. Pan London personalized budgets for rough sleepers. J Integr Care. 2014;22:23-6. https://doi.org/10.1108/JICA-07-20130024.

29. Bregman R. Utopia for realists and how we can get there. Bloomsbury paperback; 2018

30. Festinger DS, Dugosh K, Kirby KC, Seymour BL. Contingency management for cocaine treatment: cash vs. vouchers. J Subst Abus Treat. 2014:47(2):16874. https://doi.org/10.1016/j.jsat.2014.03.001.

31. Semaan S, Santibanez S, Garfein RS, Heckathorn DD, Des Jarlais DC. Ethical and regulatory considerations in HIV prevention studies employing respondent-driven sampling. Int J Drug Policy. 2009;20(1):14-27. https://doi. org/10.1016/j.drugpo.2007.12.006.

32. Jauffret-Roustide M, Le Strat Y, Couturier E, Thierry D, Rondy M, Quaglia M, et al. A national cross-sectional study among drug-users in France: epidemiology of HCV and highlight on practical and statistical aspects of the design. BMC Infect Dis. 2009:9:113.

33. Jauffret-Roustide M, Pillonel J, Weill Barillet L, Leon L, Le Strat Y, Brunet $S$ et al. Estimation de la séroprévalence du VIH et de l'hépatite $C$ chez les usagers de drogues en France. Premiers -résultats de l'enquête ANRS Coquelicot 2011. Bull Epidemiol Hebd 2013; 39-40:504-9. Available at: http://opac.invs.sante.fr/doc num.php?explnum_id=9257 (Accessed in Nov 217).

34. Cadet-Taïrou A, Dambélé S. Les CAARUD en 2010 - Analyse des rapports d'activité annuels standardisés ASA-CAARUD. OFDT 2014 Trend. Available at: http://www.ofdt.fr/publications/collections/rapports/rapports-d-etudes/ rapports-detudes-ofdt-parus-en-2014/les-caarud-en-2010-analyse-desrapports-dactivite-annuels-standardises-asa-caarud-avril-2014/. Accessed Nov 2018.

35. Heckathorn DD. Respondent-driven sampling: a new approach to the study of hidden populations. Soc Probl. 1997:44(2):174-99.

36. Islam MM, Topp L, Day CA, Dawson A, Conigrave KM. The accessibility, acceptability, health impact and cost implications of primary healthcare outlets that target injecting drug users: a narrative synthesis of literature. Int J Drug Policy. 2012;23:94-102.

37. Colvin HM, Mitchell AE, Institute of Medicine (U.S.), Committee on the Prevention and Control of Viral Hepatitis Infections, Institute of Medicine (U. S.), Board on Population Health and Public Health Practice. Hepatitis and liver cancer a national strategy for prevention and control of hepatitis B and C. Washington, DC: National Academies Press; 2010.

38. Aitken C, Moore D, Higgs P, Kelsall J, Kerger M. The impact of a police crackdown on a street drug scene: evidence from the street. Int J Drug Policy 2002; 13:189-198. Available at: http://www.popcenter.org/Responses/ police_crackdowns/PDFs/Aitken_etal_2002.pdf. Accessed in Nov 2017.

39. Cooper H, Moore L, Gruskin S, Krieger N. The impact of a police drug crackdown on drug injectors' ability to practice harm reduction: a qualitative study. Soc Sci Med. 2005;61:673-84.

40. Baraldi C, Corsi G. Niklas Luhmann: Education as a social system. Springer; 2017.

41. Makridakis S, Moleskis A. The costs and benefits of positive illusions. Front Psychol 2015;6:859. doi: https://doi.org/10.3389/fpsyg.2015.00859. eCollection 2015

42. Mauss M. The Gift: Expanded Edition. Hau; 2016.

43. Spiegel BM, Younossi ZM, Hays RD, Revicki D, Robbins S, Kanwal F. Impact of hepatitis $C$ on health related quality of life: a systematic review and quantitative assessment. Hepatology. 2005;41:790-800.

44. Batki SL1, Canfield KM, Smyth E, Ploutz-Snyder R. Health-related quality of life in methadone maintenance patients with untreated hepatitis $C$ virus infection. Drug Alcohol Depend 2009 1;101:176-182. https://doi.org/10. 1016/j.drugalcdep.2008.12.012.

45. Birkhead GS, Klein SJ, Candelas AR, O'Connell DA, Rothman JR, Feldman IS, Tsui DS, Cotroneo RA, Flanigan CA. Integrating multiple programme and policy approaches to hepatitis $C$ prevention and care for injection drug users: a comprehensive approach. Int J Drug Policy. 2007;18:417-25.

46. Evon DM, Simpson K, Kixmiller S, Galanko J, Dougherty K, Golin C, Fried MW. A randomized controlled trial of an integrated care intervention to increase eligibility for chronic hepatitis C treatment. Am J Gastroenterol. 2011;106: 1777-86. https://doi.org/10.1038/ajg.2011.219.

47. Kramer JR, Kanwal F, Richardson P, Giordano TP, Petersen LA, El-Serag HB. Importance of patient, provider, and facility predictors of hepatitis $C$ virus treatment in veterans: a national study. Am J Gastroenterol. 2011;106:48391. https://doi.org/10.1038/ajg.2010.430.

48. Mehta SH, Genberg BL, Astemborski J, Kavasery R, Kirk GD, Vlahov D, Strathdee SA, Thomas DL. Limited uptake of hepatitis C treatment among injection drug users. J Community Health. 2008;33:126-33. https://doi.org/ 10.1007/s10900-007-9083-3.

49. Midgard H, Bramness JG, Skurtveit S, Haukeland JW, Dalgard O. Hepatitis C treatment uptake among patients who have received opioid substitution treatment: a population-based study. PLoS One. 2016;11:e0166451. doi: https://doi.org/10.1371/journal.pone.0166451. eCollection 2016.

50. Lucidarme D, Duburque C, Bulois P, Filoche B. Evolution of HCV incidence in drug users in France. Epidemiol Infect. 2011;139:1287-95. https://doi.org/10. 1017/S0950268811000318.

51. van den Berg CH, Smit C, Bakker M, Geskus RB, Berkhout B, Jurriaans S, Coutinho RA, Wolthers KC, Prins M. Major decline of hepatitis $C$ virus incidence rate over two decades in a cohort of drug users. Eur J Epidemiol. 2007;22:183-93.

52. Gigerenzer G, Gaissmair W. Heuristic decision making. Annu Rev Psychol. 2011;62:451-82

53. Kramer AD, Guillory JE, Hancock JT. Experimental evidence of massive-scale emotional contagion through social networks. Proc Natl Acad Sci U S A. 2014;111:8788-90

54. Christakis NA, Fowler JH. The collective dynamics of smoking in a large social network. N Engl J Med. 2008;358:2249-58.

55. Rosenquist JN, Murabito J, Fowler JH, Christakis NA. The spread of alcohol consumption behavior in a large social network. Ann Intern Med. 2010;152: 426-33 W141.

56. SC M, Christakis NA, Fowler JH. The spread of sleep loss influences drug use in adolescent social networks. PLoS One. 2010;5:e9775. https://doi.org/10. 1371/journal.pone.0009775

57. Kenny-Walsh E. The natural history of hepatitis C virus infection. Clin Liver Dis. 2001:5:969-77.

58. European Association for Study of Liver. EASL Recommendations on Treatment of Hepatitis C 2015. J Hepatol. 2015;63:199-236. https://doi.org/ 10.1016/j.jhep.2015.03.025.

59. Grebely J, Matthews GV, Lloyd AR, Dore GJ. Elimination of hepatitis C virus infection among people who inject drugs through treatment as prevention: feasibility and future requirements. Clin Infect Dis. 2013;57:1014-20. https:// doi.org/10.1093/cid/cit377. 
60. Sidibé M, Zuniga JM, Montaner J. Leveraging HIV treatment to end AIDS, stop new HIV infections, and avoid the cost of inaction. Clin Infect Dis 2014; 59(Suppl 1):S3-S6. [PubMed: 24926030].

61. Granich RM, Gilks CF, Dye C, De Cock KM, Williams BG. Universal voluntary HIV testing with immediate antiretroviral therapy as a strategy for elimination of HIV transmission: a mathematical model. Lancet. 2009;373:48-57.

62. Thaler R, Sunstein C. Nudge improving decisions about health, health and happiness. Penguin books; 2009.

63. Halpern D. Inside the nudge unit: how small changes can make a big difference. Ebury press 206.

64. Elwyn G, Edwards A, Thompson R. Shared decision making in health care: achieving evidence-based patient choice. Oxford: Oxford University Press; 2016. https://doi.org/10.1093/acprof:oso/9780198723448.001.0001.

65. MacLellan J, Surey J, Abubakar I, Stagg HR, Mannell J. Using peer advocates to improve access to services among hard-to-reach populations with hepatitis C: a qualitative study of client and provider relationships. Harm Reduction Journal201714:76. https://doi.org/10.1186/s12954-017-0202-x

66. Festinger DS, Marlowe DB, Dugosh KL, Croft JR, Arabia PL. Higher magnitude cash payments improve research follow-up rates without increasing drug use or perceived coercion. Drug Alcohol Depend. 2008;96:128-35.

67. Crawford ND, Vlahov D. Progress in HIV reduction and prevention among injection and noninjection drug users. J Acquir Immune Defic Syndr. 2010; 55(Suppl 2):S84-7.

68. McCoy SI, Njau PF, Fahey C, Kapologwe N, Kadiyala S, Jewell NP, Dow WH, Padian NS. Cash vs. food assistance to improve adherence to antiretroviral therapy among HIV-infected adults in Tanzania: a randomized trial. AIDS. 2017:31:815-25.

69. Seal KH, Kral AH, Lorvick J, McNees A, Gee L, Edlin BR. A randomized controlled trial of monetary incentives vs. outreach to enhance adherence to the hepatitis B vaccine series among injection drug users. Drug Alcohol Depend. 2003;71:127-31.

Ready to submit your research? Choose BMC and benefit from:

- fast, convenient online submission

- thorough peer review by experienced researchers in your field

- rapid publication on acceptance

- support for research data, including large and complex data types

- gold Open Access which fosters wider collaboration and increased citations

- maximum visibility for your research: over $100 \mathrm{M}$ website views per year

At $\mathrm{BMC}$, research is always in progress.

Learn more biomedcentral.com/submissions 